\title{
Short-term cross-sensitizion of need-free sugar intake by combining sodium depletion and hypertonic $\mathrm{NaCl}$ intake
}

\author{
Bruna M. Santos, Carina A.F. de Andrade, José V. Menani, Laurival A. De Luca Jr. \\ Department of Physiology and Pathology, School of Dentistry-FOAr, São Paulo State University - UNESP, Araraquara, São Paulo, Brazil
}

\section{A R T I C L E I N F O}

\section{Article history:}

Received 16 March 2016

Received in revised form

9 June 2016

Accepted 14 July 2016

Available online 25 July 2016

\section{Keywords:}

Sodium appetite

Behavioral sensitization

Neuroplasticity

Dehydration

Thirst

Reward

\begin{abstract}
A B S T R A C T
History of sodium depletion cross-sensitizes the effects of drugs of abuse. The objective of the present study was to find out if history of sodium depletion also cross-sensitizes a natural reward such as sugar intake in the rat. Sodium depletion was induced by furosemide combined with removal of ambient sodium for $24 \mathrm{~h}$; it was repeated seven days later. The depletion was immediately followed by $0.3 \mathrm{M} \mathrm{NaCl}$ intake in a sodium appetite test (active sodium repletion). Seven days after the last depletion, hydrated and fed (need-free) sucrose-naïve animals were offered $10 \%$ sucrose in a first 2 -h sucrose test. The sucrose test was repeated once a day in a series of five consecutive days. History of sodium depletion enhanced sucrose intake in the first and second tests; it had no effect from the third to fifth sucrose test. The effect on the initial sucrose intake tests disappeared if the rats did not ingest $0.3 \mathrm{M} \mathrm{NaCl}$ in the sodium appetite test. Prior experience with sucrose intake in need-free conditions had no effect on sodium appetite. History of intracellular dehydration transiently influenced sucrose intake in the first sucrose test. We found no evidence for thirst sensitization. We conclude that history of dehydration, particularly that resulting from sodium depletion, combined to active sodium repletion, produced shortterm cross-sensitization of sucrose intake in sucrose-naïve rats. The results suggest that the crosssensitization of sucrose intake related with acquisition of sugar as a novel nutrient rather than production of lasting effects on sugar rewarding properties.
\end{abstract}

(c) 2016 Elsevier Ltd. All rights reserved.

\section{Introduction}

Sodium appetite guides a sodium-depleted animal to ingest selectively the lost nutrient (Epstein, 1991). Such example of ingestive behavior directed to a specific goal is fundamental to restore the volume of the extracellular compartment. Sodium depletion in humans occurs in response to episodic dehydration, such as that found, for example, during exercise (Hew-Butler, Verbalis, \& Noakes, 2006). In the rat, sodium depletion releases natriorexigenic hormones (angiotensin II, aldosterone) which not only induce sodium appetite, but also reorganize the brain and future behavior (Bernstein, 2003; Epstein, 1991; Hurley \& Johnson, 2013; Pereira, Menani, \& De Luca, 2010; Sakai, Fine, Epstein, \& Frankmann, 1987). One consequence of such reorganization is selective enhancement of the ingestion of sodium solutions in

\footnotetext{
* Corresponding author. Department of Physiology and Pathology, School of Dentistry, Rua Humaitá, 1680, São Paulo State University - UNESP, 14801-903, Araraquara, São Paulo, Brazil.

E-mail address: lucajr@foar.unesp.br (L.A. De Luca).
}

response to future sodium depletions (Pereira et al., 2010; Sakai et al., 1987). It corresponds to sensitization, a kind of nonassociative learning which is produced by stimulus repetition (Bernstein, 2003; Dietz, Curtis, \& Contreras, 2006; Olsen, 2011). The capacity to enhance sodium intake presumably reflects an evolutionary adaptation to environmental sodium lack, thus reducing the risks associated with extracellular dehydration (Epstein, 1991).

Similar to sodium, sugar or sweeteners added to water provides a strong reward for an animal with internal deficits such as the dehydrated rat (Cabanac, 1992; De Luca, Pereira-Derderian, Vendramini, David, \& Menani, 2010; Epstein, 1991). Rats in need-free conditions, fed and hydrated, and with no recorded history of nutritional deficits, also ingest substantial amounts of sugar (De Luca, De Souza, Yada, \& Meyer, 1999; Smith \& Sclafani, 2002). Need-free sugar intake avoids the confounding effects that lack of nutrients might have on the expression of the behavior. In other words, we have a behavior guided mainly by the rewarding properties of the goal. Rewarding or hedonic properties in conditions of plenty have a link to pathological consumption of nutrients, particularly sodium and sugar (DiNicolantonio \& Lucan, 2014; Hu \& 
Malik, 2010; Tekol, 2006).

Common reward circuits overlap in the brain potentially subserving interactions among motivated behaviors (Bernstein, 2003; Berridge, 2009; Grigson, 2002; Olsen, 2011). Cross-sensitization is one of those interactions, and sodium appetite as well as the motivation to ingest sugar cross-sensitize the effects of drugs of abuse. For example, prior experience of sodium depletion enhances the ambulatory effects of amphetamine, cocaine or morphine in rats (Acerbo \& Johnson, 2011; Clark \& Bernstein, 2004; Na, Morris, \& Johnson, 2009). Prior experience with these drugs also sensitizes sodium appetite. Equivalent reciprocal cross-sensitization occurs between sucrose intake and drugs of abuse (Avena, Rada, \& Hoebel, 2008). Thus, it is of interest to know whether the conditions that produce sodium and sugar intake also interact.

Considering that sodium depletion cross-sensitizes the effects of artificial rewards such as drugs of abuse, we asked whether history of sodium depletion also sensitizes the need-free intake of a natural reward such as sugar. We investigated whether enhanced sucrose intake follows the enhancement of sodium appetite in the rat. We also investigated whether the enhanced sucrose intake is persistent, and reciprocal sensitization occurs between sucrose intake and the production of sodium appetite. Finally, we investigated whether the ingestion of hypertonic $\mathrm{NaCl}$ in sodium appetite tests is necessary for the enhancement of sucrose intake, and whether intracellular dehydration sensitizes water and sucrose intake.

\section{Methods}

\subsection{Animals}

Male Holtzman rats from the Araraquara colony at UNESP, weighing between 270 and $280 \mathrm{~g}$ upon arrival, were used. They were maintained on a 12:12 light/dark cycle, individually housed in wire mesh suspended cages in temperature and humidity controlled rooms, and gently handled daily. Two polypropylene bottles $(100 \mathrm{ml}$ capacity with divisions to the nearest $\mathrm{ml}$ ) with stainless steel spouts, each containing water and $0.3 \mathrm{M} \mathrm{NaCl}$, were freely available per cage unless otherwise noted. Rodent food chow pellets ( $1 \%$ sodium; BioBase, Brazil) were available for the rats in a container hanging outside of each cage. The tests of all experiments began between 9 and 12 a. . m., 7 days after housing the animals with all fluids available. The protocols were approved by the Institutional Animal Care and Use Committee (CEUA FOAr, UNESP) and followed the recommendations from the National Council for the Control of Animal Experimentation (CONCEA).

\subsection{Solutions}

Sucrose and $\mathrm{NaCl}$ were dissolved in filtered tap water.

\subsection{Drugs}

Furosemide (diuretic and natriuretic, Sigma-Aldrich St. Louis, MO) was dissolved $(10 \mathrm{mg} / \mathrm{ml}$ ) in distilled water (vehicle) at a $\mathrm{pH}$ adjusted to 9.0 with $0.1 \mathrm{M} \mathrm{NaOH}$.

\subsection{Sodium depletion and sodium appetite test}

Food, $0.3 \mathrm{M} \mathrm{NaCl}$ and water was removed and the rat's cage rinsed with water. Next, sodium depletion was induced by one subcutaneous injection of furosemide $(10 \mathrm{mg} / \mathrm{ml} / \mathrm{rat})$ followed by access to only water and sodium-deficient food (powdered corn meal; $0.001 \%$ sodium and $0.33 \%$ potassium) for $24 \mathrm{~h}$. This procedure induces between 1.5 and $2.0 \mathrm{mEq}$ loss of sodium and consistent sodium appetite, which expresses or "matures" several hours after the injection of furosemide (Rowland \& Morian, 1999). A control group received subcutaneous injection of vehicle and had access to water, $0.3 \mathrm{M} \mathrm{NaCl}$ and regular food chow for $24 \mathrm{~h}$.

Twenty-four hours after the furosemide or vehicle injection, the food was removed and $0.3 \mathrm{M} \mathrm{NaCl}$ and water were offered to the animals in $0.1 \mathrm{ml}$ graduate glass burettes fitted with stainless steel spouts. The animals were warned of the presence of fluids by gentle prodding of their lips with the spout. The intake of each fluid was measured at 15, 30, 60 and 120 min (sodium appetite test). The sodium appetite test is enough to restore blood volume and the sodium lost (Sakai et al., 1987). Standard food was returned to all animals after the test and remained available with water and $0.3 \mathrm{M}$ $\mathrm{NaCl}$ until the next sodium depletion.

\subsection{Sucrose test}

As detailed in Experiments 1, 2 and 4, sucrose solution was offered daily to hydrated and fed animals beginning one week after the last sodium appetite test (Experiments 1 and 2) or thirst test (Experiment 4). The animals were naïve to sucrose solution when they entered the first sucrose intake test and the sucrose test was repeated once a day in a series of five tests.

In a slight modification of a sugar test described previously (De Luca Jr. et al., 1999), water, $0.3 \mathrm{M} \mathrm{NaCl}$ and food were removed. Immediately thereafter, $10 \%$ sucrose was offered to the animals in $0.1 \mathrm{ml}$ graduate glass burettes fitted with stainless steel spouts. The animals were warned of the presence of sucrose by gentle prodding of their lips with the spout of the drinking tube. Sucrose intake in hydrated and fed (need-free) conditions was recorded for $2 \mathrm{~h}$ (sucrose intake test). The $10 \%$ sucrose was removed at the end of the sucrose test, and food, water and $0.3 \mathrm{M} \mathrm{NaCl}$ were returned and remained available until the next test.

\subsection{Statistics}

Two-way repeated measures ANOVA was used for comparison of the means followed by the Student-Newman-Keuls post-hoc test. A probability of less than 0.05 was required for significance. Data are expressed as means \pm standard error of the mean.

\subsection{Experiment 1. Sucrose test in animals with history of sodium depletion and sodium appetite tests}

The objective of this experiment was to test if history of sodium depletion sensitizes sugar intake.

Animals were separated into two groups, those to have no history of sodium depletion (control, $\mathrm{n}=8$ ), and those to have history of sodium depletion and actively replacing their sodium loss by ingesting $0.3 \mathrm{M} \mathrm{NaCl}$ in the sodium appetite test (His/AR, $\mathrm{n}=8$ ). The His/AR group was submitted to a first sodium depletion and sodium appetite test as described above. Standard food was returned after the sodium appetite test to all animals and remained available continuously with water and $0.3 \mathrm{M} \mathrm{NaCl}$ until the next depletion induced one week later. A second sodium appetite test was performed after the second sodium depletion. One week after the second sodium depletion, standard food, $0.3 \mathrm{M} \mathrm{NaCl}$ and water were removed, and a bottle containing $10 \%$ sucrose was attached to the front of the cage thus starting the sucrose test series.

\subsection{Experiment 2. Sucrose test in animals with history of sodium depletion, but no sodium appetite test}

As shown in Results, history of sodium depletion enhanced 10\% sucrose intake in Experiment 1. The objective of Experiment 2 was to test if the experience with the ingestion of sodium in the sodium 
appetite test that followed the sodium depletion was necessary to sensitize sucrose intake.

The protocol was similar to that used in a previous work to investigate the effect of sodium intake on sodium appetite sensitization (Sakai et al., 1987). Animals were separated into two subgroups, those to have no history of sodium depletion (control, $\mathrm{n}=7$ ), and those to have history of the lost sodium replaced by the experimenter (history of passive sodium replacement or His/PR).

Food, $0.3 \mathrm{M} \mathrm{NaCl}$ and water was removed and the rat's cage rinsed with water. Next, the first sodium depletion was induced in the His/PR group by one subcutaneous injection of furosemide plus removal of ambient sodium as described for Experiment 1. The control group received subcutaneous injection of vehicle and had access to water, $0.3 \mathrm{M} \mathrm{NaCl}$ and regular food chow for $24 \mathrm{~h}$.

Twenty-four hours after the furosemide or vehicle injection, the food was removed, and both control and His/PR animals received each $2.0 \mathrm{mEq}$ of sodium distributed evenly in four separate intraperitoneal injections of $0.15 \mathrm{M} \mathrm{NaCl}$ with a sodium load equivalent to $0.5 \mathrm{mEq} / 3.5 \mathrm{ml}$ each. The four injections of $0.15 \mathrm{M} \mathrm{NaCl}$ were performed at 30-min interval. Immediately after the last injection, only water and regular food was returned for $24 \mathrm{~h}$. The bottle containing $0.3 \mathrm{M} \mathrm{NaCl}$ was returned at the end of the $24 \mathrm{~h}$ and remained available until the next sodium depletion. The second sodium depletion followed by the same type of sodium load was performed one week later in the His/PR group. One week after the second sodium depletion, food, $0.3 \mathrm{M} \mathrm{NaCl}$ and water were removed, and a bottle containing $10 \%$ sucrose was attached to the front of the cage thus starting the sucrose test series.

\subsection{Experiment 3. Sodium appetite in animals with history of sucrose intake}

The objective of this experiment was to find out if experience with the ingestion of sucrose sensitizes sodium appetite.

Sixteen animals were used. All of them had free access to water, $0.3 \mathrm{M} \mathrm{NaCl}$ and food. A third bottle contained $5 \mathrm{ml}$ of either water (control group, $\mathrm{n}=8$ ) or $10 \%$ sucrose (history of sucrose group or HisSuc, $\mathrm{n}=8$ ). Unpublished results from our laboratory showed that daily $10 \%$ sucrose intake ranges from 20 up to $40 \mathrm{ml}$. Thus, we limited the amount of $10 \%$ sucrose to $5 \mathrm{ml}$ in each access in order to guarantee the same amount of sucrose intake to all rats. In addition, as we will also see in Results, $5 \mathrm{ml}$ is within the range of the amount of $10 \%$ sucrose consumed in the first 2 -h sucrose test. The access to the third bottle was allowed about the same time of the day (beginning at noon) when the sodium appetite test and the sucrose tests were performed in Experiment 1 . The animals had access to the third bottle for seven consecutive days. All animals of the HisSuc group ingested all of the $5 \mathrm{ml}$ of $10 \%$ sucrose each day.

On the day following next to the last access to sucrose, food, $0.3 \mathrm{M} \mathrm{NaCl}$ and water was removed and the rat's cage rinsed with water. Next, sodium depletion was induced in both groups by the method of combining an injection of furosemide with removal of ambient sodium for $24 \mathrm{~h}$. Sodium appetite test began $24 \mathrm{~h}$ after the furosemide or vehicle injection.

\subsection{Experiment 4. Sucrose intake tests in animals with history of intracellular dehydration}

Sodium depletion produces extracellular dehydration. This raises the question if any kind of dehydration sensitizes sucrose intake. The objective of this experiment was to find out if history of intracellular dehydration also sensitizes sugar intake. The animals $(n=16)$ were removed from their cages and gently trained to receive an intragastric load (gavage) by infusing distilled water $(1 \mathrm{ml})$ through a polypropylene tubing connected to a syringe. The length of the tubing and the effective sodium load ( $2 \mathrm{ml}$ of $2 \mathrm{M}$ $\mathrm{NaCl}$ ) needed to induce strong hypernatremia, natriuresis, reduced plasma renin activity and thirst, were based on previous work (Pereira et al., 2002). The training began after 2 days of adaptation to the cages and was prescribed for once a day for 5 days. On the 6 th day, after food and every fluid was removed from the cage, the animals were separated into two groups, those to have no history of dehydration (control, $\mathrm{n}=8$ ), and those to have history of intracellular dehydration (HisICD, $\mathrm{n}=8$ ). The HisICD group received gavage of $2 \mathrm{M} \mathrm{NaCl}$ and the control received gavage of $0.15 \mathrm{M} \mathrm{NaCl}$. Water was offered $1 \mathrm{~h}$ after gavage and water intake was recorded for $2 \mathrm{~h}$ (thirst test). Standard food and water were available until the next intracellular dehydration and thirst test performed one week later. One week after the second thirst test, regular food chow, $0.3 \mathrm{M} \mathrm{NaCl}$ and water were removed, and a bottle containing $10 \%$ sucrose was attached to the front of the cage thus starting the fiveday sucrose test series.

\section{Results}

\subsection{Experiment 1. Sucrose test in animals with history of sodium depletion and sodium appetite tests}

The His/AR group ingested more $0.3 \mathrm{M} \mathrm{NaCl}$ than the control group in both sodium appetite tests (Table 1). It also had an enhanced $0.3 \mathrm{M} \mathrm{NaCl}$ intake in the second sodium appetite test. There was an effect of treatment $[F(1,1)=58.1 ; p<0.05]$ and test $[F$ $(1,14)=9.2 ; \mathrm{p}<0.05]$, and interaction between treatment and test $[\mathrm{F}(1,14)=7.8 ; \mathrm{p}<0.05]$.

The His/AR group ingested more water than the control in the second sodium appetite test (Table 1). There was no enhancement in water intake for both groups. There was an effect of treatment [F $(1,1)=5.8 ; \mathrm{p}<0.05]$. There was no effect of test $[\mathrm{F}(1,14)=1.5$; $\mathrm{p}=0.23]$ and no interaction between treatment and test $[\mathrm{F}$ $(1,14)=0.7 ; \mathrm{P}=0.40]$.

The His/AR group ingested more $10 \%$ sucrose than controls in the first and second sucrose tests (Fig. 1). Both groups increased their sucrose intake across tests until reaching an asymptote between the third and fifth sucrose tests (Fig. 1). There was an effect of treatment $[\mathrm{F}(1,14)=8.5 ; \mathrm{p}<0.05]$ and test $[\mathrm{F}(4,56)=29.7$; $\mathrm{p}<0.05]$, and interaction between treatment and test [F (4, $56)=6.3 ; \mathrm{p}<0.05]$.

The His/AR group ingested more $10 \%$ sucrose than controls at 15 min of the first sucrose intake test when both groups were naïve to sucrose (Table 2). There was an effect of treatment $[\mathrm{F}(1,14)=7.3$; $\mathrm{p}<0.05]$ and time $[\mathrm{F}(4,56)=9.11 ; \mathrm{p}<0.05]$, but no interaction between treatment and time $[\mathrm{F}(4,56)=2.44 ; \mathrm{p}=0.06]$.

\subsection{Experiment 2. Sucrose test in animals with history of sodium depletion, but no sodium appetite test}

His/PR $(n=7)$ and control $(n=7)$, both of which received

Table 1

Total $0.3 \mathrm{M} \mathrm{NaCl}$ and water intake $(\mathrm{ml} / 2 \mathrm{~h}$ ) of rats which actively replaced their sodium loss (His/AR) and hydrated controls during two sodium appetite tests performed at one-week interval; same animals from Fig. 1.

\begin{tabular}{|c|c|c|c|c|}
\hline \multirow[t]{2}{*}{ Group } & \multicolumn{2}{|c|}{$0.3 \mathrm{M} \mathrm{NaCl}$ intake } & \multicolumn{2}{|c|}{ Water intake } \\
\hline & $1 \mathrm{st}$ test & 2nd test & 1 st test & 2nd test \\
\hline Control & $0.7 \pm 0.3$ & $0.6 \pm 0.7$ & $0.3 \pm 0.1$ & $0.5 \pm 0.4$ \\
\hline His/AR & $10.9 \pm 2.7^{*}$ & $17.7 \pm 1.0^{*} \dagger$ & $1.3 \pm 0.1$ & $1.8 \pm 1.1^{*}$ \\
\hline
\end{tabular}

Results are reported as means \pm SEM. ${ }^{*} \mathrm{P}<0.05$ vs. Control, same sodium appetite test. $\dagger \mathrm{P}<0.05$ vs. $0.3 \mathrm{M} \mathrm{NaCl}$ intake in the 1 st sodium appetite test, same group. $\mathrm{N}=8$ /group. 


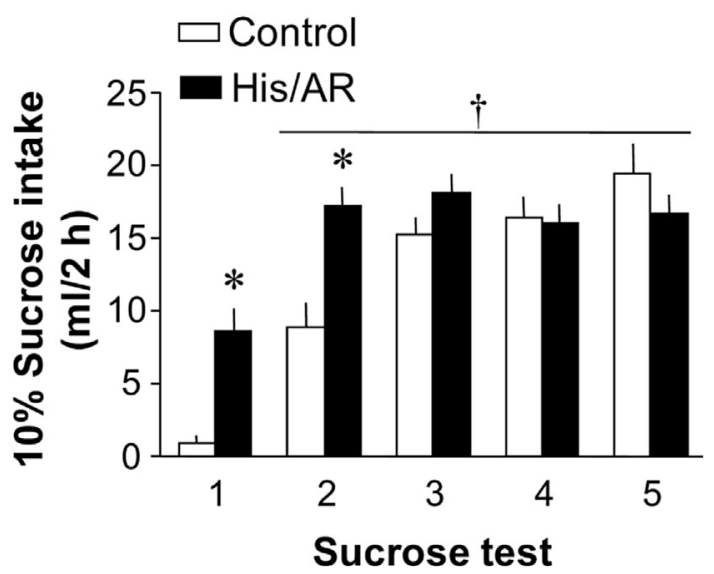

Fig. 1. Sucrose intake measured in need-free and hydrated condition at the end of the sucrose tests by rats which had history of sodium depletion and actively replaced their sodium loss (His/AR) or no history of sodium depletion (Control). $n=8 /$ group, same animals from Tables 1 and $2{ }^{*} \mathrm{P}<0.05$ vs. Control; $\dagger$ vs. Sucrose test 1 , same group.

\section{Table 2}

Non-cumulative need-free $10 \%$ sucrose intake $(\mathrm{ml})$ in the first sucrose test of sucrose-naive rats which had history of sodium depletion and active replacement of their sodium loss (His/AR), sodium depletion and passive replacement of their sodium loss (His/PR) or intracellular dehydration (HisICD), and their respective Control with no history of dehydration immediate above.

\begin{tabular}{llllll}
\hline Group & \multicolumn{5}{l}{ Time $(\mathrm{min})$} \\
\cline { 2 - 6 } & 15 & 30 & 45 & 60 & 120 \\
\hline Control & $1.9 \pm 1.0$ & $0.8 \pm 0.5$ & $0.1 \pm 0.1$ & $0.0 \pm 0.0$ & $0.0 \pm 0.0$ \\
His/AR & $5.9 \pm 1.6^{*}$ & $1.1 \pm 0.9$ & $1.3 \pm 0.8$ & $1.3 \pm 0.3$ & $0.0 \pm 0.0$ \\
Control & $3.5 \pm 1.5$ & $0.2 \pm 0.2$ & $0.0 \pm 0.0$ & $0.0 \pm 0.0$ & $0.0 \pm 0.0$ \\
His/PR & $3.4 \pm 1.1$ & $1.0 \pm 0.3$ & $0.0 \pm 0.0$ & $0.0 \pm 0.0$ & $1.9 \pm 0.9$ \\
Control & $5.0 \pm 1.2$ & $2.4 \pm 0.6$ & $0.2 \pm 0.1$ & $0.0 \pm 0.0$ & $0.1 \pm 0.0$ \\
HisICD & $8.1 \pm 1.4$ & $2.1 \pm 0.7$ & $0.2 \pm 0.1$ & $0.0 \pm 0.0$ & $0.4 \pm 0.3$
\end{tabular}

Results are reported as means \pm SEM. ${ }^{*} \mathrm{P}<0.05$ vs. respective Control immediate above. There was an interaction between treatment and time in response to history of intracellular dehydration $[\mathrm{F}(4,56)=2.8 ; \mathrm{P}<0.05]$. $\mathrm{N}=7-8$ /group.

sodium through passive sodium load instead of the sodium appetite test ingested similar amounts of $10 \%$ sucrose in the sucrose tests (Fig. 2). Both groups increased their sucrose intake across tests until reaching an asymptote between the third and fifth sucrose tests

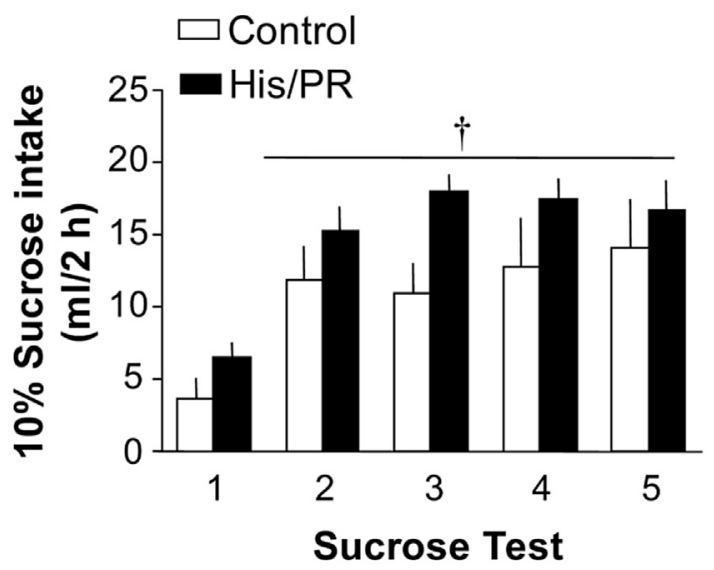

Fig. 2. Sucrose intake measured in need-free and hydrated condition at the end of the sucrose tests by rats which had history of sodium depletion and passively replaced their sodium loss (His/PR) or no history of sodium depletion (Control). $n=7$ /group, same animals from Table $2 . \dagger \mathrm{P}<0.05$ vs. Sucrose test 1 , same group.
(Fig. 2). There was an effect of test $[F(4,48)=14.8 ; \mathrm{p}<0.05]$. There was no effect of treatment $[\mathrm{F}(1,12)=3.1 ; \mathrm{p}=0.10]$ or interaction between treatment and test $[\mathrm{F}(4,48)=0.7 ; \mathrm{p}=0.6]$.

No effect of treatment $[F(1,12)=2.0 ; p=0.18]$ or interaction between treatment and test $[\mathrm{F}(4,48)=0.8 ; \mathrm{p}=0.55]$ was detected in the first sucrose test when the animals were naïve to sucrose (Table 2). There was an effect of time $[\mathrm{F}(4,48)=8.9 ; \mathrm{p}<0.05]$.

\subsection{Experiment 3. Sodium appetite in animals with history of sucrose intake}

HisSuc rats with prior experience with sucrose intake $(\mathrm{n}=8)$ and controls $(\mathrm{n}=8)$ with no such experience ingested similar amounts of $0.3 \mathrm{M} \mathrm{NaCl}$ in the sodium appetite test (Fig. 3). There was no effect of treatment $[\mathrm{F}(1,14)=2.2 ; \mathrm{p}=0.16]$, and no interaction between treatment and time $[\mathrm{F}(4,56)=0.5 ; \mathrm{p}=0.75]$. There was an effect of time $[\mathrm{F}(4,56)=25.9 ; \mathrm{p}<0.05]$.

\subsection{Experiment 4. Sucrose intake tests in animals with history of intracellular dehydration}

The HisICD group ingested more water than control in the first and second thirst tests. Repeated history of intracellular dehydration induced by gavage of $2 \mathrm{M} \mathrm{NaCl}$ slightly decreased water intake (Fig. 4). There was an effect of treatment $[\mathrm{F}(1,14)=102.1 ; \mathrm{p}<0.05]$ and test $[\mathrm{F}(1,14)=9.4 ; \mathrm{p}<0.05]$, but no interaction between treatment and test $[\mathrm{F}(1,14)=2.6 ; \mathrm{p}=0.13]$.

There was no difference in $10 \%$ sucrose intake between HisICD $(\mathrm{n}=8)$ and controls $(\mathrm{n}=8)$ in sucrose tests (Fig. 5). There was an effect of test $[\mathrm{F}(4,56)=14.7 ; \mathrm{p}<0.05]$. There was no effect of treatment $[\mathrm{F}(1,14)=0.0 ; \mathrm{P}=0.93]$ and no interaction between treatment and test $[\mathrm{F}(4,56)=2.1 ; \mathrm{p}=0.09]$.

There was an interaction between treatment and time $[\mathrm{F}(4$, $56)=2.8 ; \mathrm{p}<0.05$ ] in the first sucrose test when the animals were naïve to sucrose (Table 2). There was an effect of time [F (4, $56)=42.4 ; \mathrm{p}<0.05]$, but no effect of treatment $[\mathrm{F}(1,14)=1.3$; $\mathrm{p}=0.27]$.

\section{Discussion}

We examined the effect of history of episodic sodium depletion on need-free $10 \%$ sucrose intake of adult rats. Fed and hydrated animals performed a sucrose test once a day in a series of five tests.

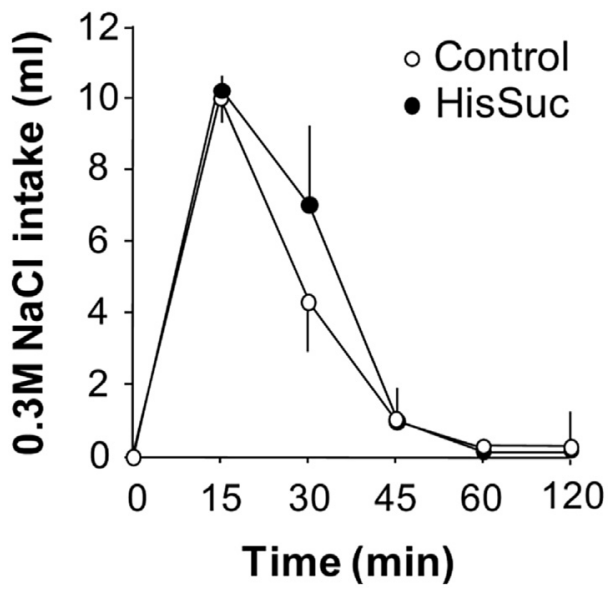

Fig. 3. Non-cumulative $0.3 \mathrm{M} \mathrm{NaCl}$ intake of sodium depleted rats which had previous experience with $10 \%$ sucrose (HisSuc) and rats with no such experience (Control). $\mathrm{n}=8$ /group. 


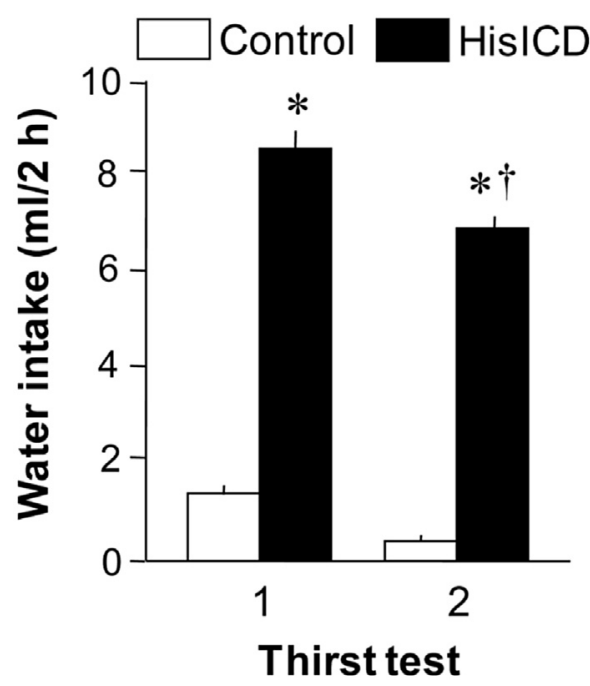

Fig. 4. Water intake in thirst tests performed by rats at one week interval in response to a gavage of $2 \mathrm{M} \mathrm{NaCl}$ given to His/ICD group. $\mathrm{n}=8$ /group; ${ }^{*} \mathrm{P}<0.05$ vs. Control; $\dagger$ vs. Thirst test 1 , same group.

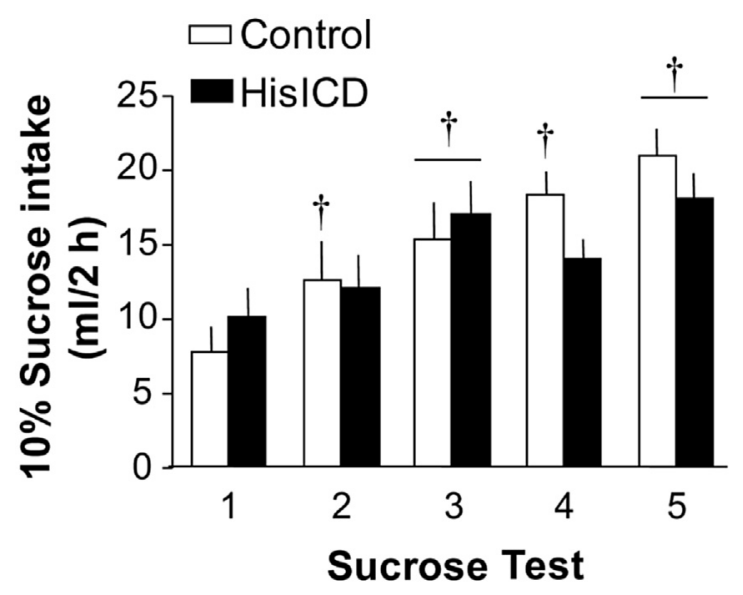

Fig. 5. Sucrose intake measured in need-free and hydrated condition at the end of the sucrose tests by rats with history of intracellular dehydration (His/ICD) or no history of dehydration (Control). $\mathrm{n}=8$ /group, same animals from Fig. $4 . \dagger \mathrm{P}<0.05$ vs. Sucrose test 1 , same group.

The series began seven days after the second and last sodium depletion. Prior experience with sodium depletion and respective sodium appetite test had a pronounced effect on need-free sucrose intake when animals were naïve to sucrose in the first sucrose test. Sucrose-naïve rats that experienced sodium depletion and actively replaced their sodium loss (His/AR group) ingested more sucrose than sucrose-naïve rats that did not have such experience (control). The difference in sucrose intake between His/AR and control group persisted in the second sucrose test. There were no differences in sucrose intake between the two groups in the remaining tests. Sucrose-naïve rats that experienced sodium depletion, but passively replaced their sodium loss (His/PR group), did not enhance their sucrose intake compared to controls.

Prior history of need-free sucrose intake did not affect the amount of $0.3 \mathrm{M} \mathrm{NaCl}$ ingested in the sodium appetite test. History of intracellular dehydration produced an interaction between treatment (gavage of hypertonic $\mathrm{NaCl}$ ) and time in the first sucrose test and no effect among tests. All groups which underwent the sucrose tests, with or without history of dehydration increased the amount of sucrose they ingested from the first to the third test (Experiments 1, 2 and 4). The amount of sucrose intake was maximal and leveled off usually after the third sucrose test.

The present results show that history of sodium depletion followed by sodium intake in the sodium appetite test (active repletion) enhanced or cross-sensitized need-free $10 \%$ sucrose in sucrose-naïve rats. History of sodium depletion induces a specific enhancement of sodium intake as compared to the ingestion of other minerals in the sodium appetite test (De Luca et al., 2010; Sakai et al., 1987). This is consistent with the selective motivation to ingest sodium by a first-time sodium-depleted animal (Epstein, 1991). The specificity of sodium intake by such animal also applies when we compare its sodium intake to the ingestion of sweetened beverages such as fruit juices and sport drinks, or even plain sucrose solutions (Epstein, 1991; Nozaki, Pereira, Moura, Menani, \& De Luca, 2002). As suggested before (McKinley, 2013), a potential aversion to sugar may also help the incentive to seek and ingest sodium because sodium-depleted rats have a reduced preference for low-sodium/high-sugar food.

The protocol we used to investigate sucrose intake sensitization, however, avoided pairing the future reward (sucrose) to history of need (sodium depletion and its physiological consequences) and satisfaction of the need (sodium intake), similarly to previous works done with drugs of abuse (Acerbo et al., 2011; Clark \& Bernstein, 2004; Na et al., 2009). We offered the to-be sensitized reward, sucrose, to naïve animals for the first time, in need-free state, several days after the animals experienced sodium depletion and respective sodium appetite test. Thus, in spite of the specificity of sodium appetite, we may assume that history of sodium depletion combined to active sodium repletion crosssensitized need-free sucrose intake.

The cross-sensitization was of a short-term type, and likely resulted from the integration of signals produced by sodium depletion and the consequent ingestion of $0.3 \mathrm{M} \mathrm{NaCl}$. Thus, it did not follow exactly the same rules that apply for the sensitization of sodium intake. History of sodium depletion increased the responses of the rat to $10 \%$ sucrose in the first two days, but repeating the experience with the sugar removed this effect. In contrast, an early work found that sodium appetite sensitization lasts much longer, at least four months after a single sodium depletion (Sakai et al., 1987). Sensitization of daily-need free sodium intake may also well persist up to at least one month in young-adult rats (De Luca et al., 2010). This suggests that the common reward circuit shared by different motivated behaviors (Grigson, 2002; Olsen, 2011) relates to a mechanism that avoids persistent crosssensitization between sodium depletion and a natural reward such as sucrose intake.

Avoiding persistent cross-sensitization could explain similar situation in which human infants with congenital adrenal hyperplasia of the salt wasting or sodium depletion form have increased taste preference for sodium, but not sweets (Kochli, TenenbaumRakover, \& Leshem, 2005). Sodium depletion may have produced an initial cross-sensitization in their early childhood, but the repeated experience with sugar would have masked the evidence for sensitization of sugar intake, and perhaps its sweet taste, when they were still infants.

A similar hypothesis for a mechanism that limits crosssensitization between sodium depletion and a natural reward such as sugar applies to water intake in rats. It is worth noting that the methods that produce and sensitize sodium intake also produce, but do not sensitize, water intake (De Luca et al., 2010; Pereira et al., 2010; Sakai et al., 1987; present work). Offering water and $\mathrm{NaCl}$ solution simultaneously to the sodium depleted rat may sometimes deceive us into finding false water intake sensitization (Pereira et al., 2010). Allowing the rat first satiate thirst prior to 
ingesting sodium clearly confirms the difficulty to sensitize water intake (De Luca et al., 2010; Hurley \& Johnson, 2013). We may thus attribute the increased water intake found in particular circumstances as secondary to the sensitized, increased ingestion of a hypertonic $\mathrm{NaCl}$ solution. Thirst is a motivation naturally entitled to share common reward mechanisms with sodium appetite because of their essential and complementary roles for the maintenance of bodily-fluid balance. Nevertheless, evidence points to a mechanism that restricts sensitization of water intake. It is possible that a similar mechanism limited a persistent cross-sensitization between sodium depletion and sucrose intake. This raises an open question of whether such restrictive mechanism extends to crosssensitization among natural rewards in general.

The ingestion of salt in response to sodium depletion had a significant influence on future sucrose intake. Sodium replacement by intraperitoneal injections of isotonic $\mathrm{NaCl}$ instead of ingestion of $0.3 \mathrm{M} \mathrm{NaCl}$ during the sodium appetite test precluded the increase in $10 \%$ sucrose intake. Similar procedure to replace sodium does not affect sodium appetite sensitization (Sakai et al., 1987). Moreover, the hormones angiotensin II and aldosterone produced in response to sodium depletion are necessary and sufficient to sensitize sodium appetite (Sakai et al., 1987). In contrast, the hormones or any other physiological consequence of sodium depletion seemed not enough to cross-sensitize sucrose intake. The experience of spontaneously ingesting $0.3 \mathrm{M} \mathrm{NaCl}$ in daily need-free conditions, prior to sodium depletions, might include a putative role for taste (Dietz et al., 2006). Yet, given the differences found in sucrose intake between His/AR and control group in the present work, whatever the role for a previous experience with the taste or ingestion of salt in need-free conditions, it failed to produce a main effect on sucrose intake without the aid of sodium depletion. Different from what happens to sodium appetite sensitization, the enhanced sucrose intake must have resulted from sodium depletion combined to taste, post-ingestive stimuli, or both, produced by the $0.3 \mathrm{M} \mathrm{NaCl}$ intake in the sodium appetite test.

The cross-sensitization between sodium depletion/active repletion and need-free sucrose was not reciprocal. Reciprocal cross-sensitization supports the concept of a common reward circuit for motivated behaviors (Clark \& Bernstein, 2004; Grigson, 2002; Olsen, 2011). Our results do not contradict such concept, but suggest that sodium depletion cross-sensitized sucrose intake under different rules. As mentioned above, episodic repeated sodium depletions, each satisfied by a respective sodium appetite test, cross-sensitize the effects of drugs of abuse in need-free conditions (Clark \& Bernstein, 2004; Na et al., 2009; Acerbo et al., 2011). In contrast, hunger or sugar hyperphagia cross-sensitizes the effects of drugs of abuse under chronic restricted access to food (Avena et al., 2008; Carr, 2002). In spite of the differences among protocols, the cross-sensitization with the effects of the drugs of abuse is reciprocal for all the three conditions, sodium depletion (Clark \& Bernstein, 2004; Na et al., 2009; Acerbo et al., 2011), hunger (Carr, 2002) and sugar hyperphagia (Avena et al., 2008). The failure to find reciprocity in the present work suggests that the mechanism of cross-sensitization between sodium depletion and need-free sucrose intake followed some rules that deviate from those which control cross-sensitization between sodium depletion and drugs of abuse.

The rules to produce cross-sensitization might differ at the level of brain mechanisms. The sucrose test model used here assumes that need-free sugar intake activates reward systems directly by ascending sensory information raised from activation of taste or post-ingestive receptors (Lin, Amodeo, Arthurs, \& Reilly, 2012; Smith \& Sclafani, 2002; de Araujo et al., 2008). A corollary is that need-free sugar intake is independent from physiological alterations (e.g. metabolic deficits) equivalent to those produced by sodium depletion. Similar assumption is apparently implicit in the cross-sensitization studies of sodium depletion and drugs of abuse (Clark \& Bernstein, 2004; Na et al., 2009; Roitman, Na, Anderson, Jones, \& Bernstein, 2002). However, further scrutiny on the role of physiological factors might be wanted for at least two reasons. First, it is remarkable that cross-sensitization between drugs of abuse and consumption of macronutrients require a schedule of chronic food deprivation (Avena et al., 2008; Carr, 2002). The binge protocol to induce sugar hyperphagia being particularly relevant to the present results (Avena et al., 2008). We do not know if such protocol would cross-sensitize with the effects of sodium depletion. Second, the drugs of abuse which cross-sensitize their effects with that of sodium depletion have the potential to influence both energetic and water metabolism as well as interact with hormones that produce neuroplasticity (Acerbo \& Johnson, 2011; Grell, Christensen, \& Fjalland, 1985; Han, Kelly, Fellingham, \& Conlee, 1996; Marchand, 1970; McBride \& Flynn, 2007; Mello \& Mendelson, 1997; Nencini, Valeri, \& Morrone, 1990). Thus, another possible level of difference in the rules of crosssensitization, not mutually exclusive to that dependent on sensory afference to the brain, could reside in physiological or metabolic, and hormonal alterations. It would be of interest to confirm if and at which level an unidirectional rule of cross-sensitization between two natural rewards apply, particularly if one of the rewards is need-free, but the answer requires further research which goes beyond the objectives of the present work.

The results suggest that history of intracellular dehydration might also have somehow cross-sensitized sucrose intake in the HisICD group. In spite of the absence of treatment effect across sucrose tests, there was an interaction between treatment and time within the first sucrose test of sucrose-naïve rats. If crosssensitization occurred, it lasted even less than that produced by sodium depletion. The experiment with intracellular dehydration also has implications for two aspects of sensitization we have mentioned in previous paragraphs. On the one hand, the possibility of cross sensitization between intracellular dehydration and sucrose intake might support the concept of common reward circuit. On the other hand, the failure of history of intracellular dehydration to enhance water intake in thirst tests reinforces the hypothesis of a mechanism that limits thirst sensitization.

We also found that sodium depletion/active repletion-induced cross-sensitization did not alter the increase in sucrose intake expected to occur across sucrose tests. Repeated sucrose tests performed in need-free state enhanced sucrose intake until reaching an asymptote both in animals with experience of dehydration (sodium depletion or gavage of hypertonic $\mathrm{NaCl}$ ) and controls with no such experience. Such kind of asymptotic behavior shown by all groups presumably reflects neophobia for novel tastes, even rewarding tastes such as those produced by sugar or sweeteners (Lin et al., 2012). Previous work suggests that repeated exposure to the sweet taste of saccharin increases its palatability thereby its ingestion (Lin et al., 2012). In addition to its rewarding sweet taste, we may also consider a rewarding post-ingestive effect of sucrose (de Araujo et al., 2008). Thus, increased palatability combined to post-ingestive reward produced in the initial sucrose tests explains the asymptote of sucrose intake we found in both test (His/AR, His/ PR or HisICD) and control animals. A convergence between what happens to neophobia and the cross-sensitized sucrose intake might occur, but further work is necessary to find out if animals sensitized by sodium depletions perceived the first ingestion of sucrose more rewarding (e.g. more palatable).

In summary, the results suggest that the cross-sensitizing effects of history of dehydration in general - and the one produced by sodium depletion/active repletion in particular - on sugar intake relates with the ingestion of sugar as a novel nutrient rather than 
production of persistent effects on sugar rewarding properties. We found that history of sodium depletion combined to immediate and subsequent $0.3 \mathrm{M} \mathrm{NaCl}$ intake produced a short-term cross-sensitization of future need-free sucrose intake in sucrose-naïve rats. The effect on sucrose intake occurred during the initial phase of intermittent daily access to sucrose by animals that were naïve to sucrose in the first sucrose test. Such an effect resulted from one or more combined mechanisms. Possible mechanisms involved the experience of satiating a motivated need (through ingestion of sodium) and the internal products of extracellular dehydration, plus, at least in part, the activation of a common reward circuit.

\section{Acknowledgements}

The authors thank SP Barbosa, RC Queiroz and S Fóglia for technical assistance; SAD Malavolta and C Molina for secretarial assistance; AV Oliveira and M dos Santos for animal care. This research was supported by public funding from Conselho Nacional de Desenvolvimento Científico e Tecnológico (CNPq, 301296/ 2009-0; 301939/2013-6) and Fundação de Amparo à Pesquisa do Estado de São Paulo (FAPESP, 11/50770-1). BM Santos was recipient of FAPESP [2011/23696-5] and CNPq [136364/2010-1] undergraduate fellowship and CAPES graduate fellowship in the Joint UFSCar-UNESP Graduate Program in Physiological Sciences (PIPGCF UFSCar-UNESP).

\section{References}

Acerbo, M. J., \& Johnson, A. K. (2011). Behavioral cross-sensitization between DOCAinduced sodium appetite and cocaine-induced locomotor behavior. Pharmacology Biochemistry and Behavior, 98(3), 440-448.

de Araujo, I. E., Oliveira-Maia, A. J., Sotnikova, T. D., Gainetdinov, R. R., Caron, M. G., Nicolelis, M. A. L., et al. (2008). Food reward in the absence of taste receptor signaling. Neuron, 57(6), 930-941.

Avena, N. M., Rada, P., \& Hoebel, B. G. (2008). Evidence for sugar addiction: Behavioral and neurochemical effects of intermittent, excessive sugar intake. Neuroscience and Biobehavioral Reviews, 32(1), 20-39.

Bernstein, I. L. (2003). Interaction between natural motivational systems and those which respond to drugs of abuse. Appetite, 41(3), 333-334.

Berridge, K. C. (2009). "Liking" and "wanting" food rewards: Brain substrates and roles in eating disorders. Physiology and Behavior, 97(5), 537-550.

Cabanac, M. (1992). Pleasure: The common currency. Journal of Theoretical Biology, 155(2), 173-200.

Carr, K. D. (2002). Augmentation of drug reward by chronic food restriction: Behavioral evidence and underlying mechanisms. Physiology and Behavior, 76(3), 353-364.

Clark, J. J., \& Bernstein, I. L. (2004). Reciprocal cross-sensitization between amphetamine and salt appetite. Pharmacology Biochemistry and Behavior, 78(4), 691-698.

De Luca, L. A., De Souza, R. L. N., Yada, M. M., \& Meyer, E. W. (1999). Sedation and need-free salt intake in rats treated with clonidine. Pharmacology Biochemistry and Behavior, 62(4), 585-589.

De Luca, L. A., Pereira-Derderian, D. T. B., Vendramini, R. C., David, R. B., \& Menani, J. V. (2010). Water deprivation-induced sodium appetite. Physiology and Behavior, 100(5), 535-544.

Dietz, D. M., Curtis, K. S., \& Contreras, R. J. (2006). Taste, salience, and increased NaC ingestion after repeated sodium depletions. Chemical Senses, 31(1), 33-41.

DiNicolantonio, J. J., \& Lucan, S. C. (2014). The wrong white crystals: Not salt but sugar as aetiological in hypertension and cardiometabolic disease. Open Heart 1(1), e000167.

Epstein, A. N. (1991). Neurohormonal control of salt intake in the rat. Brain Research Bulletin, 27(3-4), 315-320.
Grell, S., Christensen, J. D., \& Fjalland, B. (1985). Morphine antidiuresis in conscious rats: Contribution of vasopressin and blood pressure. Acta Pharmacologica et Toxicologica, 56(1), 38-43.

Grigson, P. S. (2002). Like drugs for chocolate: Separate rewards modulated by common mechanisms? Physiology and Behavior, 76(3), 389-395.

Han, D. H., Kelly, K. P., Fellingham, G. W., \& Conlee, R. K. (1996). Cocaine and exercise: Temporal changes in plasma levels of catecholamines, lactate, glucose, and cocaine. The American Journal of Physiology, 270(3 Pt 1), E438-E444.

Hew-Butler, T., Verbalis, J. G., \& Noakes, T. D. (2006). Updated fluid recommendation: Position statement from the international marathon medical directors association (IMMDA). Clinical Journal of Sport Medicine: Official Journal of the Canadian Academy of Sport Medicine, 16(4), 283-292.

Hu, F. B., \& Malik, V. S. (2010). Sugar-sweetened beverages and risk of obesity and type 2 diabetes: Epidemiologic evidence. Physiology \& Behavior, 100(1), 47-54.

Hurley, S. W., \& Johnson, A. K. (2013). Dissociation of thirst and sodium appetite in the furo/cap model of extracellular dehydration and a role for N-methyl-Daspartate receptors in the sensitization of sodium appetite. Behavioral Neuroscience, 127(6), 890-898.

Kochli, A., Tenenbaum-Rakover, Y., \& Leshem, M. (2005). Increased salt appetite in patients with congenital adrenal hyperplasia 21-hydroxylase deficiency. American Journal of Physiology, Regulatory, Integrative and Comparative Physiology, 288(6), R1673-R1681.

Lin, J. Y., Amodeo, L. R., Arthurs, J., \& Reilly, S. (2012). Taste neophobia and palatability: The pleasure of drinking. Physiology and Behavior, 106(4), 515-519.

Marchand, C. (1970). The diuretic and antidiuretic effect of morphine sulphate in rats. Proceedings of the Society for Experimental Biology and Medicine. Society for Experimental Biology and Medicine (New York, N.Y.), 133(4), 1303-1306.

McBride, S. M., \& Flynn, F. W. (2007). Centrally administered vasopressin crosssensitizes rats to amphetamine and drinking hypertonic NaCl. American Journal of Physiology, Regulatory, Integrative and Comparative Physiology, 293(3), R1452-R1458.

McKinley, M. J. (2013). Adaptive appetites for salted and unsalted food in rats: Differential effects of sodium depletion, effects of sodium depletion, DOCA, and dehydration. American Journal of Physiology, Regulatory, Integrative and Comparative Physiology, 304(12), R1149-R1160.

Mello, N. K., \& Mendelson, J. H. (1997). Cocaine's effects on neuroendocrine systems: Clinical and preclinical studies. Pharmacology, Biochemistry, and Behavior, 57(3), 571-599.

Na, E. S., Morris, M. J., \& Johnson, A. K. (2009). Behavioral cross-sensitization between morphine-induced locomotion and sodium depletion-induced salt appetite. Pharmacology Biochemistry and Behavior, 93(4), 368-374.

Nencini, P., Valeri, P., \& Morrone, L. A. (1990). Dapiprazole, a selective alpha-1 adrenoceptor antagonist, inhibits diuresis but not polydipsia produced by amphetamine in rats. Brain Research Bulletin, 25(5), 765-767.

Nozaki, P. N., Pereira, D. T. B., Moura, F. V., Menani, J. V., \& De Luca, L. A. (2002). Ingestion of hypertonic $\mathrm{NaCl}$ vs. palatable drinks by sodium-depleted rats. Physiology and Behavior, 75(4), 443-448.

Olsen, C. M. (2011). Natural rewards, neuroplasticity, and non-drug addictions. Neuropharmacology, 61(7), 1109-1122.

Pereira, D. T. B., Menani, J. V., \& De Luca, L. A. (2010). FURO/CAP: A protocol for sodium intake sensitization. Physiology and Behavior, 99(4), 472-481.

Pereira, D. T. B., Vendramini, R. C., David, R. B., Nozaki, P. N., Menani, J. V., \& De Luca, L. A. (2002). Isotonic NaCl intake by cell-dehydrated rats. Physiology and Behavior, 76(4-5), 501-505.

Roitman, M. F., Na, E., Anderson, G., Jones, T. A., \& Bernstein, I. L. (2002). Induction of a salt appetite alters dendritic morphology in nucleus accumbens and sensitizes rats to amphetamine. The Journal of Neuroscience: The Official Journal of the Society for Neuroscience, 22(11), RC225.

Rowland, N. E., \& Morian, K. R. (1999). Roles of aldosterone and angiotensin in maturation of sodium appetite in furosemide-treated rats. American Journal of Physiology, Regulatory, Integrative and Comparative Physiology, 276(5 Pt 2), R1453-R1460.

Sakai, R. R., Fine, W. B., Epstein, A. N., \& Frankmann, S. P. (1987). Salt appetite is enhanced by one prior episode of sodium depletion in the rat. Behavioral Neuroscience, 101(5), 724-731.

Smith, J. C., \& Sclafani, A. (2002). Saccharin as a sugar surrogate revisited. Appetite, 38(2), 155-160.

Tekol, Y. (2006). Salt addiction: Aa different kind of drug addiction. Medical Hypotheses, 67(5), 1233-1234. 